\title{
Antiretroviral Therapy among HIV-1 Infected Female Sex Workers in Benin: A Comparative Study with Patients from the General Population
}

\author{
Souleymane Diabaté ${ }^{1,2}$, Djimon Marcel Zannou ${ }^{3}$, Nassirou Geraldo ${ }^{4}$, Annie Chamberland ${ }^{1}$, \\ Jocelyn Akakpo ${ }^{3}$, Carin Ahouada ${ }^{3}$, Marguerite Massinga Loembé ${ }^{\text {, Sévérin Anagonou }}{ }^{3}$, \\ Annie Claude Labbé ${ }^{6}$, Michel Alary ${ }^{2}$, Cecile Tremblay ${ }^{1}$
} ${ }^{1}$ Centre de Recherche du Centre Hospitalier de l'Université de Montréal, Montréal, Canada; ${ }^{2}$ URESP, Centre de recherche FRSQ du
CHA universitaire de Québec, Québec, Canada; ${ }^{3}$ Centre National Hospitalier Universitaire de Cotonou, Cotonou, Bénin; ${ }^{4}$ Dispensaire
IST, Centre de Santé de Cotonou I, Cotonou, Bénin; ${ }^{5}$ Institut de Médecine Tropicale d'Anvers, Anvers, Belgique; ${ }^{6}$ Université de
Montréal, Hôpital Maisonneuve-Rosemont, Montréal, Canada.

Email: dsouleym@hotmail.com

Received June $7^{\text {th }}, 2011$; revised June $24^{\text {th }}, 2011$; accepted July $4^{\text {th }}, 2011$.

\begin{abstract}
Objective: The aim of this study conducted in Benin was to compare HIV-1 infected female sex workers (FSW) and patients from the general population (GP) to see whether there was a difference in adherence level, mortality rate and immuno-virologic response to antiretroviral therapy (ART). Methods: Fifty-tree FSW and 318 patients from the GP were recruited and followed for at least one year. We compared both cohorts according to poor-adherence (taking $<95 \%$ of the pills), CD4 count increase, undetectable viral load (VL; $\leq 50$ copies $/ \mathrm{mL})$ and crude mortality rate. We constructed a multivariate regression model to assess factors associated with undetectable VL. Results: During the first year, the proportion of FSW with poor-adherence was significantly higher than that of the GP patients (19.3\% versus $7.5 \% ; p<0.0001)$ and median gain in CD4 count among FSW was slightly lower $\left(103 / \mathrm{mm}^{3}\right.$ versus $\left.129 / \mathrm{mm}^{3} ; p=0.085\right)$. In the multivariate model (including CD4 at ART initiation and the sub-cohort i.e. FSW vs GP patients), duration under ART $(p=0.003)$ as well as CD4 count at enrolment in the study $(p<0.0001)$ and good-adherence $(0.057)$ were independently associated with undetectable VL. When adherence was withdrawn from this model, there was a borderline significant association between detectable VL and being a FSW $(p=0.074)$. The crude mortality rate was 1.11 per 100 persons-years among the GP patients and 4.65 per 100 persons-years among FSW. Conclusion: Response to ART was lower among FSW compared to GP patients, as a result of poorer adherence. Specific behavioural interventions are needed to improve adherence and response to ART among FSW.
\end{abstract}

Keywords: ART, FSW, Adherence

\section{Introduction}

In 2009, more than 22.5 million people (including approximately 1.8 million new infections) were living with HIV in sub-Saharan Africa [1]. In this region, female sex workers (FSW) and their clients have played an important role in the extension of the epidemic to the general population (GP) [2,3]. Consequently, both groups have been targeted by various prevention interventions like promotion of condom use and diagnosis and treatment of sexually transmitted infections (STI). These interventions aiming at reducing HIV acquisition as well as transmission have, indeed, contributed to an increase in condom use and a decline in STI prevalence [4-6]. Nonetheless, in sub-Saharan Africa, HIV prevalence among FSW is still 3 to 4 times higher than among the general population (19\% versus 5.2\%) [7]. It is therefore important to consider improving access to antiretroviral therapy (ART) for FSW and to scale-up other preventive interventions shown to be effective [8]. According to recent findings on the efficacy of ART for HIV prevention [9], providing early ART to HIV-infected female sex workers has the potential to have a considerable impact on HIV transmission to their clients and therefore contribute to HIV prevention in the general population. Despite the increasing 
number of treated patients in sub-Saharan Africa, little is known about access to ART among FSW [1]. The objective of this prospective study conducted in Cotonou (Benin) was to compare HIV-1 infected FSW and patients from the GP to see whether there was a difference in adherence level, mortality rate and immuno-virologic response to ART.

\section{Methods}

From September 2008 to September 2010, we consecutively enrolled and followed $371 \mathrm{HIV}-1$ infected adults (aged $\geq 18$ years) receiving a first-line ART composed of two nucleoside reverse transcriptase inhibitors (NRTI) plus a non-nucleoside reverse transcriptase inhibitor (NNRTI) or a protease inhibitor (PI)): Fifty-three (53) patients were FSW recruited at the Dispensaire IST (medical center dedicated since 1989 to routine check-up and STI treatment among FSW) and 318 patients were from the GP. The GP patients were recruited at the Centre de traitement ambulatoire (CTA) du Centre national hospitalier universitaire de Cotonou (reference center for HIV treatment in Benin). After enrolment in the study, FSW were seen every 3 months and patients from the GP every 4 months (in accordance with existing follow-up visits' chronograms in each study center). World Health Organisation (WHO) clinical stages III/IV or CD4 count $\leq 200 / \mathrm{mm}^{3}$ were the main conditions for ART initiation in Benin. The study was approved by the research ethics committees of the Centre hospitalier de l'Université de Montréal and the CHA universitaire de Québec in Canada, and of the Ministry of Health in Benin. An informed consent form was signed by all the participants.

Adherence counselling was routinely done in each study center and the interviewers were all trained on the study procedures. Adherence to ART was assessed through self-report of missed doses: taking $<95 \%$ of the pills prescribed during the four days preceding each visit was defined as poor adherence [10]. Adherence to ART and CD4 count (Flow cytometry) were measured at study entry and at each follow-up visit. For patients who initiated treatment before enrolment in the study, baseline CD4 count, age and WHO clinical classification were collected from the medical files. Viral load (VL, bDNA) was performed for all patients only at study entry. Descriptive comparisons using chi-square or Fisher's exact tests (proportions) and Student's t test (means) were performed to compare both cohorts according to baseline characteristics. A univariate model was performed for each potential determinant of undetectable VL. All variables with a $P$ value $\leq 0.10$ were included in a multivariate model to determine those independently associated with undetectable VL ( $\leq 50$ copies $/ \mathrm{mL})$. Data were ana- lysed with SAS version 9.2 (SAS Institute, Cary, NC, USA).

\section{Results}

At ART initiation, the median age (IQR) in both groups was comparable: thirty nine $(39 ;(33-43))$ years for the FSW and 37 (31 - 43) years for the patients from the GP $(\mathrm{p}=0.179)$. The majority of the GP patients were females $(62.3 \%)$ and Beninese $(91 \%)$. The FSW were from Benin $(30 \%)$ and neighbouring countries like Togo $(30 \%)$, Ghana (24\%) and Nigeria (15\%). The proportion of non educated FSW was higher $(35.9 \%$ versus $22.6 \%$; $\mathrm{p}=0.034)$. At enrolment in the study, median duration (IQR) under ART was $21(4-38)$ months for the GP patients and $4.3(0-24.3)$ months for the FSW $(\mathrm{p}<$ $0.003)$. At ART initiation, most of the GP patients $(73.9 \%)$ were classified as WHO clinical stages III/IV as compared with FSW $(22.6 \%, \mathrm{p}<0.0001)$.

The NRTI mostly prescribed was stavudine $(71.7 \%$ for the GP patients and $77.4 \%$ for the FSW) whereas the NNRTI mostly prescribed was efavirenz for patients from the GP (72.4\%) and nevirapine for FSW (73.6\%; Table 1). During follow-up, 19 ART modifications due to side effects were observed among the GP patients (none among the FSW). These treatment modifications occurred after a median (IQR) duration under ART of 32 (7.8 - 53.8) months. In $18 / 19$ cases (94.7\%), stavudine was replaced by zidovudine because of lipodystrophy (lipoatrophy: 11/18, lipoatrophy plus lipohypertrophy: $3 / 18)$ and peripheral neuropathy (4/18). There were 13 (8.6\%) switches to second-line ART in the GP patients. The Median time (IQR) from initiation to switch to second-line was 37.6 (27.9 - 49.0) months. Among the FSW, we observed $4(7.5 \%)$ switches. The 17 switches occurred after availability of the VL measured at study entry and were mostly related to insufficient viro-immunologic response $(88.2 \%)$.

During the first year, poor adherence, as assessed at follow-up visits, varied between $11.8 \%$ and $27.3 \%$ among the FSW and between $5.9 \%$ and $10.6 \%$ among the GP patients. Overall, the proportion of FSW with poor adherence was significantly higher than that of the GP patients $(19.3 \%$ versus $7.5 \% ; \mathrm{p}<0.0001)$. There was no difference in adherence levels according to gender in the GP patients. The main reasons for missing doses reported by the FSW were being out of home (58.6\%) and running out of pills (20.7\%).

At ART initiation, median CD4 count $/ \mathrm{mm}^{3}$ (IQR) among the GP patients and the FSW was similar 115 (53 - 184) versus 134 (67 - 175, $p=0.141)$, respectively. During the first year of treatment, we observed an increase in CD4 count $/ \mathrm{mm}^{3}$ among the FSW, but, median (IQR) 
Table 1. Antiretroviral combinations in 53 HIV-1 infected female sex workers and 318 patients from the general population, Cotonou, Benin.

\begin{tabular}{|c|c|c|}
\hline Antiretroviral combinations & General population (number, \%) & Female sex workers (number, \%) \\
\hline Stavudine + Lamivudine + Efavirenz & $164(51.6)$ & $9(17)$ \\
\hline Zidovudine + Lamivudine + Efavirenz & $65(20.4)$ & $5(9.4)$ \\
\hline Lamivudine + Didanosine + Efavirenz & $2(0.6)$ & - \\
\hline Stavudine + Lamivudine + Nevirapine & $63(19.8)$ & $32(60.4)$ \\
\hline Zidovudine + Lamivudine + Nevirapine & $23(7.2)$ & $7(13.2)$ \\
\hline Stavudine + Lamivudine + Indinavir & $1(0.3)$ & - \\
\hline Total & 318 & 53 \\
\hline
\end{tabular}

gain 103 (25 - 178) was to some extent lower as compared with the GP patients 129 (75 - 232; p = 0.085; Figure 1). At study entry, $57 \%$ of the GP patients and $34 \%$ of the FSW had undetectable VL.

After adjustment for CD4 count at ART initiation, treatment duration, as well as CD4 count and good adherence at enrolment in the study were independently associated with undetectable VL (Table 2; model 1). When we withdrew adherence from this model, being recruited from the GP was found to be related to undetectable VL (Table 2, model 2). The crude mortality rate was 1.11 per 100 person-years (3 deaths/270.33 person-years) among the GP patients and 4.65 per 100 person-years (2 deaths/43 person-years) among FSW.

In a sub-group analysis, we observed that from ART initiation to year-3, CD4 count has increased progressively among the GP patients ( $\mathrm{t}$ test for trend $=18.92 ; \mathrm{p}$ $<0.0001$; Figure 1). Median (IQR) gain in CD4 count during the same period was $263(196-345) / \mathrm{mm}^{3}$. There was no difference in CD4 progression according to the NNRTI used (nevirapine or efavirenz, $p=0.585$ ). In addition, the proportion of the GP patients recruited with undetectable VL was similar irrespective of the NNRTI prescribed (nevirapine, 60.5\% and efavirenz, 55.7\%; $\mathrm{p}=$ 0.366). Due to their small number, FSW were excluded from these sub-group analyses concerning follow-up $>1$ year and the relation between the NNRTI regimen (nevirapine versus efavirenz) and immuno-virologic response.

\section{Discussion}

Irrespective of their HIV status, FSW from Cotonou and its suburbs are requested to undergo each month a routine medical visit. This preventive approach is an opportunity for healthcare workers for early detection and treatment of STI and other morbidities. It also allows testing for HIV and prescription of cotrimoxazole prophylaxis if better clinical conditions at ART initiation among FSW needed. As a consequence, there was a trend towards as compared with the GP patients: the proportion of subjects with advanced WHO clinical stages (III/IV) was lower among FSW. This observation, in agreement with previous results showing a decrease in STI prevalence among FSW in Benin, supports the importance of maintaining and reinforcing preventive efforts towards high-risk groups $[5,11]$.

As previously described, stavudine (the most prescribed NRTI in our study populations) was the main cause for most of the first-line ART modifications due to peripheral neuropathy and lipodystrophy [12,13]. This known stavudine toxicity profile has led the WHO to recommend less toxic agents such as tenofovir or zidovudine [14]. Lipodystrophy did not lead to regimen modification in FSW probably because of a lower median duration under ART [13,15]. Most of the switches to second-line ART were based on VL measured at enrolment in the study. This is a paradox since all (except one) patients with elevated viral load also had immunologic failure and could have been switched to second-line therapy earlier, based on this sole parameter. It appeared that clinicians were less confident if they had to base their decision of switching to second-line on CD4 counts only [16]. This conservative attitude needs to be addressed by training activities aiming at strengthening physicians' prescription abilities but also by facilitating access to VL, in particular for patients with insufficient immune response [17].

In general, FSW have higher challenges for treatment adherence because of their mobility motivated by many reasons such as looking for new clients and avoiding harassment from the police, from the brothel owners or sometimes from their boyfriends. To prevent the negative impact of this social instability on adherence level, care 


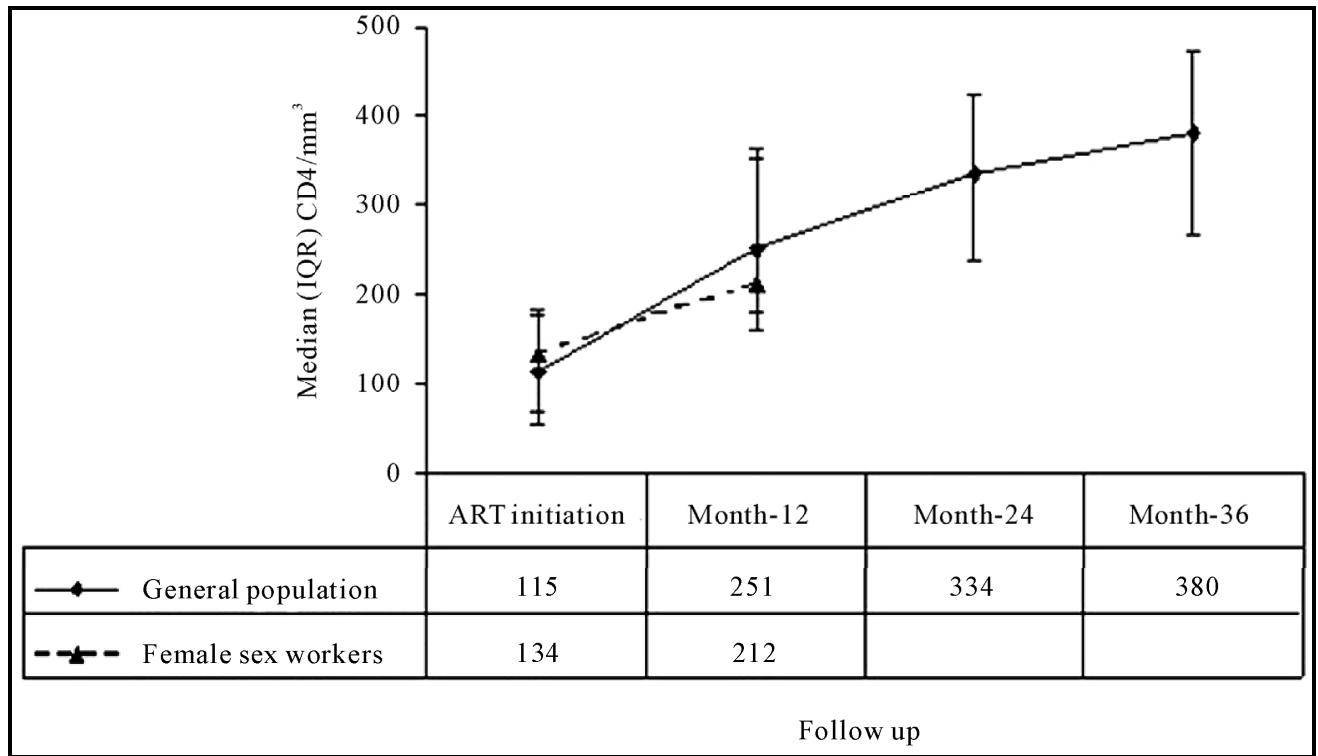

Figure 1. Comparative CD4 counts increase in HIV-1 treated female sex workers and patients from the general population, Cotonou, Benin. Note: Numbers represent median CD4 counts $/ \mathbf{m m}^{3}$; For patients who initiated antiretroviral therapy before study entry, CD4 counts were obtained from their medical file; Due to limited data, FSW were excluded from analyses of more than 12 months.

Table 2. Multivariate regression model assessing factors associated with undetectable viral load among HIV-1 treated female sex workers and patients from the general population, Cotonou, Benin.

\begin{tabular}{|c|c|c|c|c|c|c|}
\hline \multirow[b]{3}{*}{ Variables } & \multicolumn{6}{|c|}{ Undetectable viral load ( $\leq 50$ copies $/ \mathrm{mL}$ ) } \\
\hline & \multicolumn{3}{|c|}{ Model 1: with adherence } & \multicolumn{3}{|c|}{ Model 2: without adherence } \\
\hline & OR & $95 \% \mathrm{CI}$ & $\mathrm{P}$ value & OR & $95 \% \mathrm{CI}$ & $P$ value \\
\hline $\mathrm{CD} 4$ count $\geq 350 / \mathrm{mm}^{3 \dagger}$ & 3.01 & $1.87-5.11$ & $<0.0001$ & 3.01 & $1.87-5.06$ & $<0.0001$ \\
\hline Duration under ART $\geq 6$ months & 2.3 & $1.31-3.92$ & 0.003 & 2.5 & $1.46-4.20$ & 0.008 \\
\hline Being recruited from the general population & 1.2 & $0.54-2.84$ & 0.612 & 1.9 & $0.94-3.97$ & 0.074 \\
\hline Taking at least $95 \%$ of the pills prescribed ${ }^{\dagger}$ & 2.3 & $0.97-5.27$ & 0.057 & - & - & - \\
\hline
\end{tabular}

${ }^{\dagger}$ Measured at enrolment (as was viral load); OR, Odds ratio; CI, Confidence interval; Both models were adjusted for CD4 count at ART initiation.

givers at Dispensaire IST have decided to prescribe whenever possible the most simplified ART regimen available: a twice per day fixed-dose combination composed of stavudine, lamuvidine and nevirapine (Triomune; Cipla, Mumbai, India) [18-20]. This explains why FSW were more exposed to Nevirapine. Nonetheless, the majority of our study patients who claimed poor adherence were FSW and self-reported main reasons for not taking pills were indeed related to instability: being out of home and running out of pills. Strategies aiming at maximising adherence to antiretroviral therapy among FSW should tackle inherent factors like social instability.

During the first year of treatment, CD4 count recovery among FSW and GP patients reached expected levels [21]. However, this recovery was to some extent lower among FSW. In addition, viral load suppression was stronger in GP patients (Table 2; model 2), whereas the crude mortality rate was higher in the FSW. Response to ART appeared then to be less good among FSW. This difference in response to ART was neither due to gender nor to a difference of efficacy between nevirapine and efavirenz: our subgroup analyses (GP patients), in accordance with previous results, showed that response to ART did not vary significantly between subjects treated by either of these drugs [22-24]. Moreover, we have recently shown (based on the same study cohorts) that the 
prevalence of primary resistance to ART was similar in both the FSW and the GP patients [25]. Then, the remaining factor that could explain the difference in response to ART between both groups is cumulative adherence levels. Indeed, in our multivariate regression model 2, being a GP patient was associated with undetectable VL. In model 1, this association was dissipated by good adherence i.e. taking at least $95 \%$ of the pills prescribed by the doctors. Specific behavioural interventions targeting FSW are therefore needed to improve adherence and response to ART: as primary drug resistance has been found to be elevated in this population, poor adherence resulting in partial viral suppression can rapidly jeopardize ART benefits [10,25-26]. Our study has some limitations including the small number of HIV-1-treated FSW and the short-term follow-up. However, our data provides important insights on the feasibility and also on the challenges of ART delivery in the FSW population in sub-Saharan Africa.

In conclusion, response to ART was not as good among female sex workers, compared with patients from the general population, as a result of poorer adherence. Efforts to improve response to ART among female sex workers should target inherent factors impacting adherence such as social instability. In addition, large size and long term follow-up studies are needed to better analyse the determinants and the extent to which response to ART among female sex workers is different from response among the general population patients.

\section{Acknowledgements}

We would like to thank the patients and the staff at the Dispensaire IST and the Centre de traitement ambulatoire du Centre national hospitalier universitaire de Cotonou. We also thank the staff at the Centre de recherche du Centre hospitalier universitaire de Montréal and the Unité de recherche en santé des populations du Centre de recherche FRSQ du CHA universitaire de Québec for their administrative and technical assistance.

\section{REFERENCES}

[1] UNAIDS, "UNAIDS Report on the Global AIDS Epidemic", UNAIDS, Geneva, 2010. http://www.unaids.org/globalreport/documents/20101123 _GlobalReport_full_en.pdf

[2] Z. Mukandavire and W. Garira, "Effects of Public Health Educational Campaigns and the Role of Sex Workers on the Spread of HIV/AIDS among Heterosexuals," Theoretical Population Biology, Vol. 72, No. 3, 2007, pp. 346365. doi:10.1016/j.tpb.2007.07.002

[3] C. M. Lowndes, M. Alary, H. Meda, C. A. Gnintoungbe, L. Mukenge-Tshibaka, C. Adjovi, et al., "Role of Core and Bridging Groups in the Transmission Dynamics of HIV and STIs in Cotonou, Benin, West Africa," Sexually Transmitted Infections, Vol. 78, Supplement 1, 2002, pp. S69- S77. doi:10.1136/sti.78.suppl 1.169

[4] P. D. Ghys, M.O. Diallo, V. Ettiegne-Traore, K. Kale, O. Tawil, M. Carael, et al., "Increase in Condom Use and Decline in HIV and Sexually Transmitted Diseases among Female Sex Workers in Abidjan, Côte d'Ivoire, 1991-1998," AIDS, Vol. 16, No. 2, 2002, pp. 251-258. doi:10.1097/00002030-200201250-00015

[5] C. M. Lowndes, M. Alary, A. C. Labbe, C. Gnintoungbe, M. Belleau, L. Mukenge, et al., "Interventions among Male Clients of Female Sex Workers in Benin, West Africa: An Essential Component of Targeted HIV Preventive Interventions," Sexually Transmitted Infections, Vol. 83, No. 7, 2007, pp. 577-581. doi:10.1136/sti.2007.027441

[6] R. Hayes, D. Watson-Jones, C. Celum, J. van de Wijgert and J. Wasserheit, "Treatment of Sexually Transmitted Infections for HIV Prevention: End of the Road or New Beginning?” AIDS, Vol. 24, Suppl. 4, 2010, pp. S15-S26. doi:10.1097/01.aids.0000390704.35642.47

[7] UNAIDS, “AIDS Epidemic Update,” UNAIDS, Geneva, 2009.

http://data.unaids.org/pub/Report/2009/jc1700_epi_updat e_2009_en.pdf

[8] N. Malunguza, S. Mushayabasa, C. Chiyaka and Z. Mukandavire, "Modelling the Effects of Condom Use and Antiretroviral Therapy in Controlling HIV/AIDS among Heterosexuals, Homosexuals and Bisexuals," Computational and mathematical methods in medicine, Vol. 11, No. 3, 2010, pp. 201-22. doi:10.1080/17486700903325167

[9] Editorial, "HIV Treatment as Prevention-It Works," Lancet, Vol. 377, No. 9779, 2011, p.1719. doi:10.1016/S0140-6736(11)60713-7

[10] N. Ford, M. Darder, T. Spelman, E. Maclean, E. Mills and A. Boulle, "Early Adherence to Antiretroviral Medication as a Predictor of Long-Term HIV Virological Suppression: Five-Year Follow Up of an Observational Cohort," PLoS One, Vol. 5, No. 5, 2010, p. e10460. doi:10.1371/journal.pone.0010460

[11] P. Vickerman, F. Ndowa, N. O'Farrell, R. Steen, M. Alary and S. Delany-Moretlwe, "Using Mathematical Modelling to Estimate the Impact of Periodic Presumptive Treatment on the Transmission of Sexually Transmitted Infections and HIV among Female Sex Workers," Sexually Transmitted Infections, Vol. 86, No. 3, 2010, pp. 163-168. doi:10.1136/sti.2008.034678

[12] J. van Griensven, L. de Naeyer, T. Mushi, S. Ubarijoro, D. Gashumba, C. Gazille and R. Zachariah, "High Prevalence of Lipoatrophy among Patients on Stavudine-Containing First-Line Antiretroviral Therapy Regimens in Rwanda," Transactions of the Royal Society of Tropical Medicine and Hygiene, Vol. 101, No. 8, 2007, pp. 793798. doi:10.1016/j.trstmh.2007.02.020

[13] S. Mercier, N. F. Gueye, A. Cournil, A. Fontbonne, N. 
Copin, I. Ndiaye, et al., "Lipodystrophy and metabolic Disorders in HIV-1-Infected Adults on 4- to 9-Year Antiretroviral Therapy in Senegal: A Case-Control Study," Journal of Acquired Immune Deficiency Syndrom, Vol. 51, No. 2, 2009, pp. 224-230.

[14] World Health Organisation, "Antiretroviral Therapy for HIV Infection in Adults and Adolescents. Recommadation for a Public Health approach: 2010 Revision," WHO, Geneva, 2010.

http://whqlibdoc.who.int/publications/2010/97892415997 64 eng.pdf

[15] D. M. Zannou, L. Denoeud, K. Lacombe, D. AmoussouGuenou, J. Bashi, J. Akakpo, et al., "Incidence of Lipodystrophy and Metabolic Disorders in Patients Starting Non-Nucleoside Reverse Transcriptase Inhibitors in Benin," Antiviral Therapy, Vol. 14, No. 3, 2009, pp. 371380 .

[16] O. Keiser, H. Tweya, P. Braitstein, F. Dabis, P. MacPhail and A. Boulle, "Mortality after Failure of Antiretroviral Therapy in Sub-Saharan Africa," Tropical medicine \& international health, Vol. 15, No. 2, 2010, pp. 251-258. doi:10.1111/j.1365-3156.2009.02445.X

[17] O. Keiser, H. Tweya, A. Boulle, P. Braitstein, M. Schecter and M. W. Brinkhof, "Switching to Second-Line Antiretroviral Therapy in Resource-Limited Settings: Comparison of Programmes with and without Viral Load Monitoring," AIDS, Vol. 23, No. 14, 2009, pp. 1867-1874. doi:10.1097/QAD.0b013e32832e05b2

[18] E. J. Mills, J. B. Nachega, D. R. Bangsberg, S. Singh, B. Rachlis, P. Wu, et al., "Adherence to HAART: A Systematic Review of Developed and Developing Nation Patient-Reported Barriers and Facilitators," PLoS Medicine, Vol. 3, No. 11, 2006, p. e438.

[19] C. Protopopescu, F. Raffi, P. Roux, J. Reynes, P. Dellamonica, B. Spire, et al., "Factors Associated with NonAdherence to Long-Term Highly Active Antiretroviral Therapy: A 10 Year Follow-Up Analysis with Correction for the Bias Induced by Missing Data," The Journal of Antimicrobial Chemotherapy, Vol. 64, No. 3, 2009, pp. 599-606. doi:10.1093/jac/dkp232
[20] A. D. Bouhnik, M. Chesney, P. Carrieri, H. Gallais, J. Moreaum J. P. Moatti, et al., "Nonadherence among HIV-Infected Injecting Drug Users: The Impact of Social Instability," Journal of acquired immune deficiency syndromes, Vol. 31, Supplement 3, 2002, pp. S149-S153. doi:10.1097/00126334-200212153-00013

[21] D. Nash, M. Katyal, M. W. Brinkhof, O. Keiser, M. May R. Hughes, et al., "Long-Term Immunologic Response to Antiretroviral Therapy in Low-Income Countries: A Collaborative Analysis of Prospective Studies," AIDS, Vol. 22, No. 17. 2008, pp. 2291-2302.

[22] P. de Beaudrap, J. F. Etard, F. N. Gueye, M. Gueye, R. Landman, P. Girard, et al., "Long-Term Efficacy and Tolerance of Efavirenz- and Nevirapine-Containing Regimens in Adult HIV Type 1 Senegalese Patients," AIDS research and human retroviruses, Vol. 24, No. 6, 2008, pp. 753-760. doi:10.1089/aid.2007.0295

[23] S. Toure, B. Kouadio, C. Seyler, M. Traore, N. Dakoury-Dogbo, J. Duvignac, et al., "Rapid Scaling-Up of Antiretroviral Therapy in 10,000 Adults in Côte d'Ivoire: 2-Year Outcomes and Determinants," AIDS, Vol. 22, No. 7, 2008, pp. 873-882. doi:10.1097/QAD.0b013e3282f768f8

[24] E. Nicastri, S. Leone, C. Angeletti, L. Palmisano, L. Sarmati, A. Chiesi, et al., "Sex Issues in HIV-1-Infected Persons during Highly Active Antiretroviral Therapy: A Systematic Review," The Journal of antimicrobial chemotherapy, Vol. 60, No. 4, 2007, pp. 724-732. doi: $10.1093 / \mathrm{jac} / \mathrm{dkm} 302$

[25] A. Chamberland, S. Diabaté, M. Sylla, Y. S. Anagounou, N. Geraldo, D. M. Zannou, et al., "HIV-1 Primary Drug Resistance Prevalence and Transmission in Cotonou, Benin," 5th Conference of French Speeking Countries on HIV/ AIDS, Casablanca, March 2010, p. 110.

[26] D. L. Hanson, C. Adje-Toure, N. Talla-Nzussouo, P. Eby, M. Y. Borget, L. Y. Kouadio, et al., "HIV Type 1 Drug Resistance in Adults Receiving Highly Active Antiretroviral Therapy in Abidjan, Côte d'Ivoire," AIDS Research and Human Retroviruses, Vol. 25, No. 5, 2009, pp. 489 495. doi:10.1089/aid.2008.0273 